\title{
Cantes de ida y vuelta en la formación sobre la gestión del hábitat. Aprendizajes y confluencias entre el Paraná y el Guadalquivir
}

\section{Roundtrip songs in the habitat formation and social management. Findings and nodes between the Parana and Guadalquivir river}

\author{
Esteban de Manuel Jerez, ${ }^{*}$ M. ${ }^{a}$ Bernabela Pelli** y José M. ${ }^{a}$ López Medina***
}

Fecha de recepción: 08-05-2017 - Fecha de aceptación: 07-09-2017

Hábitat y Sociedad (ISSN 2173-125X), n. ${ }^{\circ}$ 10, noviembre de 2017, pp. 323-334.

http://dx.doi.org/10.12795/HabitatySociedad.2017.i10.18

\section{Abstract}

The training of professionals capable of designing and following processes of the Habitat Production and Social Management implies the acquisition of a systemic integrated conceptual framework of theoretical conceptual knowledge and methodological action guidelines. Moving from a specialized, disciplinary and object-oriented approach to an interdisciplinary perspective and an approach focused on process design is the educational problem on which we have been experimenting in groups with teams of teachers from four Latin American and Spanish universities located around the Parana and Guadalquivir rivers. Roundtrip songs describes the experience and results in terms of our team's designs learning of postgraduate courses jointly taught in Resistencia, Montevideo and Seville.

\section{Key words}

Habitat production and social management; Reflective professional training; Postgrad; Reflection-action; Case study

\section{Resumen}

La formación de profesionales capaces de diseñar y acompañar procesos de Producción y Gestión Social del Hábitat implica la adquisición de un marco conceptual sistémico e integrado de conocimientos teóricoconceptuales y de pautas metodológicas para la acción. Pasar de una perspectiva especializada, disciplinar y de un enfoque centrado en el diseño de objetos, a una perspectiva interdisciplinar y un enfoque centrado en el diseño de procesos es el problema educativo sobre el que venimos experimentando de forma cooperativa equipos docentes de cuatro universidades, latinoamericanas y españolas, ${ }^{1}$ ubicadas en torno al Paraná y el Guadalquivir. Cantes de ida y vuelta describe la experiencia y los resultados en términos de aprendizajes de nuestros equipos en el diseño de cursos de posgrado impartidos de forma conjunta en Resistencia, Montevideo y Sevilla.

\section{Pallabras clave}

Producción y gestión social del hábitat; Formación de profesionales reflexivos; Posgrado; Reflexión-acción; Estudio de casos

\footnotetext{
* Dr. Arquitecto. Grupo ADICI HUM-810, Profesor titular de la E.T.S. Arquitectura de la Universidad de Sevilla, España. C.e.: edemanuel@us.es.

** Arquitecta Instituto de Investigación y Desarrollo en Vivienda (IIDVi), Facultad de Arquitectura y Urbanismo de la Universidad Nacional del Nordeste (UNNE). Resistencia, Chaco, Argentina. C.e.: mariabernabelapelli@hotmail.com.

*** Dr. Arquitecto. Grupo ADICI HUM810, El Taller de Hábitat 4, S.C.A. C.e.: josemalo.es@gmail.com.

1 Equipos: Español, Grupo ADICI Universidad de Sevilla y grupo GIEST Universidad de Pablo de Olavide. Argentino, Universidad Nacional del Nordeste. Facultad de Arquitectura y Urbanismo. Instituto de Investigación y Desarrollo en Vivienda (IIDVi). Uruguayo, Universidad de la República. Facultad de Arquitectura.
} 


\section{Introducción}

Algunos palos flamencos se llaman cantes de ida y vuelta o cantes hispanoamericanos, porque nacieron de la influencia mutua entre las culturas de una y otra orilla del océano Atlántico, a través de los movimientos migratorios y las relaciones que se establecieron entre América y España. De un modo similar esta reflexión recoge la experiencia de una colaboración entre equipos docentes de Sevilla (España), Resistencia (Argentina) y Montevideo (Uruguay). Pero a la ida y vuelta entre continentes también se suma la ida y vuelta entre los profesores y los distintos grupos de alumnos, un proceso de retroalimentación de la reflexión en la acción, que ha supuesto igualmente un aprendizaje mutuo y que ha permitido ir perfeccionando el método docente de un curso a otro.

El subprograma HABITED (Tecnología para viviendas de interés social) del programa CYTED (Ciencia y Tecnología para el Desarrollo), promovido por la AECID, ha permitido articular redes latinoamericanas e ibéricas de investigadores e investigadoras socialmente comprometidas con el hábitat social. Por otro lado, la red Universitaria Latinoamericana de Cátedras de Vivienda (ULACAV) ha hecho lo propio con la docencia de grado. Ellas han propiciado los antecedentes de colaboración que crearon las bases de cooperación interuniversitaria en torno a la formación en producción y gestión social del hábitat. ${ }^{2}$

Nuestros respectivos equipos de trabajo venían desarrollando, de forma paralela, experiencias en las que las funciones docente e investigadora se alimentan de las experiencias de extensión universitaria en cada contexto. El proyecto de consolidación urbana del asentamiento chabolista de Jnane Aztout (2005-2013), en el marco del Plan Villes Sans Bidonvilles del Ministerio del Hábitat de Marruecos, propició el encuentro y la cooperación de nuestros equipos. El contexto de intervención (extensión universitaria) creó la necesidad de formación de los profesionales implicados (educación, aprendizaje-servicio) y de profundizar en las herramientas de comprensión de la realidad sobre la que intervenir (investigación en la acción participativa) (De Manuel, 2017).

Esta experiencia sirvió de catalizadora para el diseño del Máster en Gestión Social del Hábitat (De Manuel et al., 2009), al que fueron invitados como docentes los profesionales del equipo argentino del IIDVi. Esta intensificación de la cooperación entre los equipos propició el reto de acometer conjuntamente el diseño de cursos de formación de posgrado. Oferta cuya finalidad fue propiciar la incorporación de herramientas conceptuales y metodológicas para el diseño e implementación de procesos de transformación socio-espacial adecuadas a los distintos contextos, basándonos como premisas en la participación y concertación de los actores involucrados (técnicos, políticos y vecinales), la interdisciplinariedad, la intersectorialidad, la multiescalaridad y la sustentabilidad.

A partir de esta base compartida coincidimos en diseñar conjuntamente cursos de formación de posgrado centrados en prácticas de intervención, alimentadas teórica y metodológicamente, siguiendo la secuencia que empleamos en la intervención participativa en hábitat social:

- aproximación y reconocimiento de una necesidad habitacional, formulada como tema-problema sobre el que intervenir;

- diagnóstico integral, participativo y prospectivo; 
- formulación de una propuesta de transformación de la realidad habitacional.

PROCESO desde la NECESIDAD HABITACIONAL hacia la formulación de un proyecto integral para su SOLUCIÓN

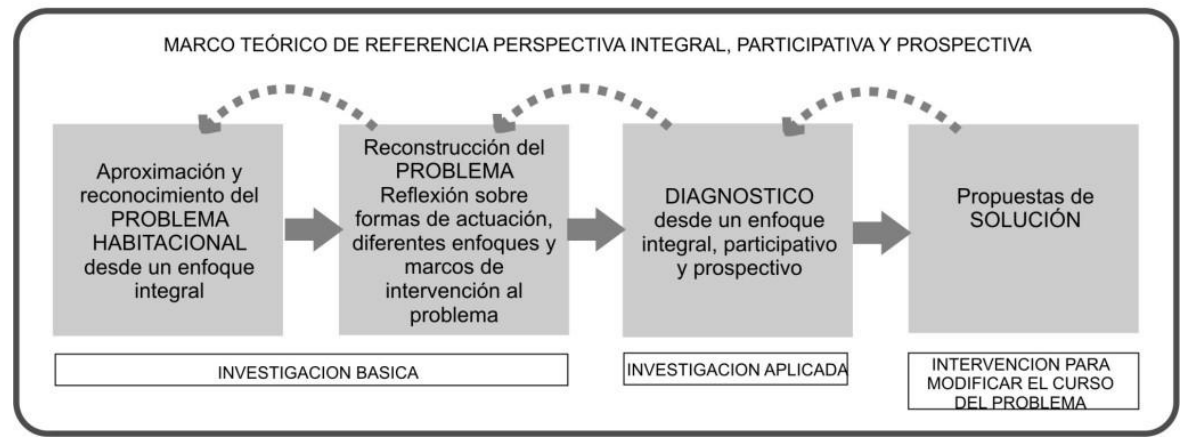

\section{Experiencias de cooperación universitaria para} formación en posgrado en Producción y Gestión Social del Hábitat entre el Paraná y el Guadalquivir

El marco institucional que dio cobertura a la experiencia fue el programa de cooperación interuniversitaria de la Agencia de Cooperación Internacional para el Desarrollo de España. Dicho marco propició dos proyectos consecutivos centrados en el diseño de cursos de posgrado en producción y gestión social del hábitat a cargo de los tres equipos de las universidades públicas de Sevilla, Resistencia y Montevideo.

El primer proyecto nos permitió diseñar tres cursos autónomos, a cargo de cada uno de los tres equipos (2008-09). Se impartirían de forma itinerante en Resistencia, Montevideo y Sevilla, con un formato presencial intensivo de una semana de duración cada uno de ellos. En cada sede se impartieron los tres cursos con matriculación independiente. El proyecto sirvió a su vez para impulsar la revista científica Hábitat y Sociedad y para apoyar la estancia como investigadora de una doctoranda sevillana en la Universidad de la República de Uruguay para desarrollar su tesis sobre el cooperativismo uruguayo de vivienda.
Figura 1. Proceso de intervención social participativa en hábitat social. Fuente: Elaboración propia.

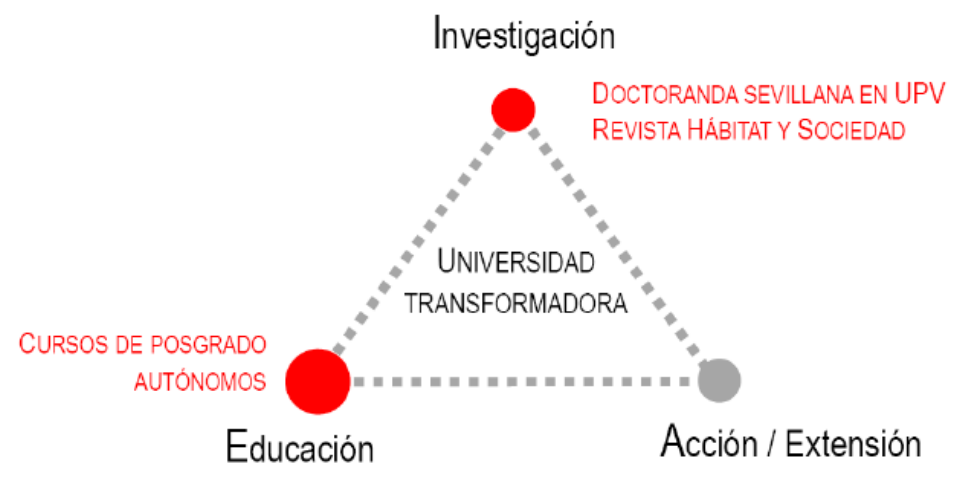

El encuentro anual de la red ULACAV en Resistencia (2009) nos permitió coordinarnos y formular el segundo proyecto (2010-2011). Se organizó un nuevo bloque formativo de posgrado en el que se mantuvo la autonomía organizativa y docente de los equipos, pero los tres cursos se ofertaron como un único paquete agrupado.

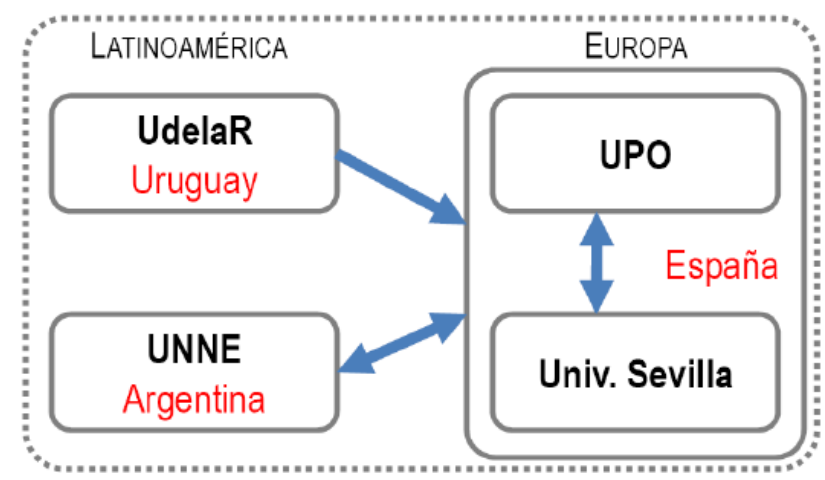

Figura 2. Construcción cooperativa de la base del triángulo de la universidad transformadora a través de la relación entre diseño de cursos de posgrado autónomos e investigación en Producción y Gestión Social del Hábitat. Fuente: Elaboración propia. 

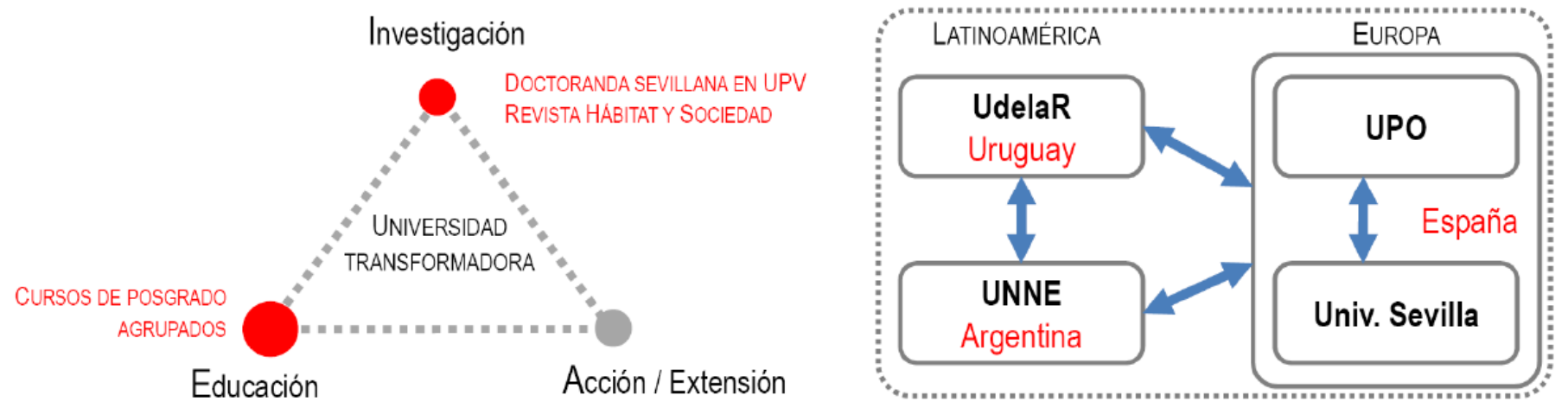

Figura 3. Construcción cooperativa de la base del triángulo de la universidad transformadora a través de la relación entre diseño de cursos de posgrado agrupados e investigación en Producción y Gestión Social del Hábitat. Fuente: Elaboración propia.

Figura 4. Evolución del diseño cooperativo de la oferta de cursos formativos de posgrado en Producción y Gestión Social del Hábitat. Fuente: elaboración propia.
Este segundo proyecto nos permitió profundizar la coordinación, desbordar el marco del proyecto de cooperación inicialmente diseñado y alimentar los procesos formativos de los respectivos equipos.

El siguiente esquema nos servirá para localizar y justificar el relato de tres casos que responden a tres estadios diferentes: el curso de Montevideo en mayo de 2011, como caso de curso autónomo a cargo de un equipo (GSH); el curso de Resistencia en octubre de 2011, como caso de mayor conjunción entre equipos coordinado por uno de ellos (IIDVi) e interesantes logros en cuanto a síntesis de los aportes a cargo de los cursantes en los trabajos finales; y el curso de Salto en junio de 2012, diseñado e impartido por un equipo mixto formado por integrantes de los tres grupos (GSH-IIDVi-UPV).

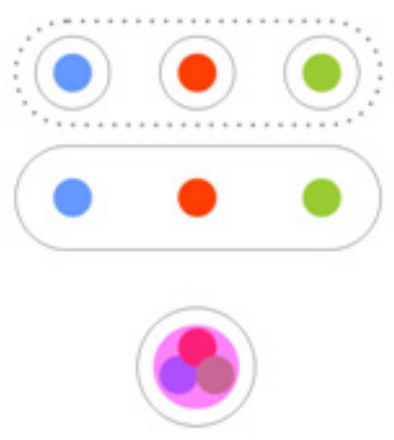

\section{Convocatoria simultánea de tres cursos independientes CASO I: Montevideo Curso unitario de tres módulos, uno por equipo docente CASO II: Resistencia} Curso integrado por los tres equipos CASO III: Salto

\section{Caso I. Tanteo en Montevideo}

El primer caso que presentamos es el de un curso diseñado de forma autónoma por uno de los equipos, en este caso, el grupo de la Universidad de Sevilla, e impartido en Montevideo (2011).

El curso llevó por título "Introducción a la producción y gestión social del hábitat”. Nos propusimos rediseñar la estructura metodológica para dar respuesta a la principal carencia detectada en la primera edición, celebrada en 2010 en Resistencia: la falta de un espacio para aplicar los conceptos y herramientas desarrollados en las sesiones expositivas.

Hicimos un diseño semipresencial, con un seminario de cinco sesiones presenciales consecutivas durante una semana, de lunes a viernes, estructuradas con una primera parte expositiva y una segunda de taller. La parte de taller consistiría en la realización de una práctica en equipo de formulación de un proyecto de Gestión Social del Hábitat. Era importante que los alumnos durante las cinco sesiones de taller desarrollaran el proyecto con el acompañamiento de los docentes. 
Los talleres presenciales desarrollados durante el seminario permitieron definir el tema-problema habitacional, hacer un pre-diagnóstico y un mapa de actores y formular una primera aproximación a la estrategia de actuación. Los equipos continuaron desarrollando de forma no presencial el tema y entregaron sus proyectos siguiendo el marco y la metodología de la gestión social del hábitat presentada en el curso.

De la evaluación del curso por los estudiantes extraemos como aprendizaje la validación del diseño del curso ("Creo que la sistematicidad del método de trabajo que han planteado ordena mucho a la hora de abordar procesos tan complejos como los que implican la gestión social del hábitat. Este método clarifica los actores, las acciones, etc."), la pertinencia de introducir el estudio de casos de Producción y Gestión Social del Hábitat desarrolladas por los docentes ("La presentación de estudios de caso para el tratamiento de los diferentes temas del curso resulta sumamente pedagógica y esclarecedora"), la constatación por parte de los estudiantes de que el curso les aporta herramientas para el desempeño profesional ("Lo que me llevo para aplicar es el triángulo POLIS-URBS-CIVITAS, me pareció muy interesante”) y la importancia de que la composición de los estudiantes propicie el trabajo interdisciplinario ("Me resultó muy gratificante trabajar con compañeros de otras disciplinas, reflexionar y discutir sobre un mismo temaproblema desde diferentes formaciones").

Podemos concluir que la estructura del curso funcionó bien para un grupo interdisciplinario compuesto por profesionales sociales y de la arquitectura, involucrados, en su gran mayoría, en programas de intervención en hábitat social.

\section{Caso II. Convergencia en Resistencia}

El curso de posgrado "Modelos de Gestión Habitacional en Iberoamérica"’ significó un siguiente paso en la integración entre los tres equipos, en el que se elaboró una oferta formativa conjunta, con la coordinación académica del grupo argentino. Se logró la convergencia de los distintos aportes en una propuesta común, adecuada a las necesidades y demandas del contexto geográfico en el que se impartió.

A partir del esquema general, que como estrategia pedagógica simula un proceso de intervención en un problema habitacional determinado, se logró la convergencia de los distintos aportes, tanto teórico-conceptuales, como metodológicos, en un programa único, organizado en tres módulos, lo que marcaba el aporte diferenciado de los distintos grupos y los diferentes contextos de actuación, en un proceso pedagógico común. Cada grupo participó desde su capital cognitivo más fuerte, completando de esta manera un conjunto de aportes de importante riqueza para los cursantes. La mayor síntesis de esta convergencia teórico-metodológica, la consiguieron hacer los participantes del curso, que lograron trabajos finales donde plasmaron los contenidos adquiridos de una manera coherente y creativa.

Los participantes, mayoría de profesionales de distintas disciplinas, pertenecientes a diversas instituciones gubernamentales de las ciudades de Corrientes y Resistencia, cuyas incumbencias están relacionadas con la temática del curso (Institutos de Vivienda, equipos de Programas de Mejoramiento Barrial-ProMeBa, Universidad y otros), tuvieron la oportunidad de intercambiar con sus compañeros, que les posibilitó sacar conclusiones y generar propuestas de cambio al interno de sus lugares de trabajo.

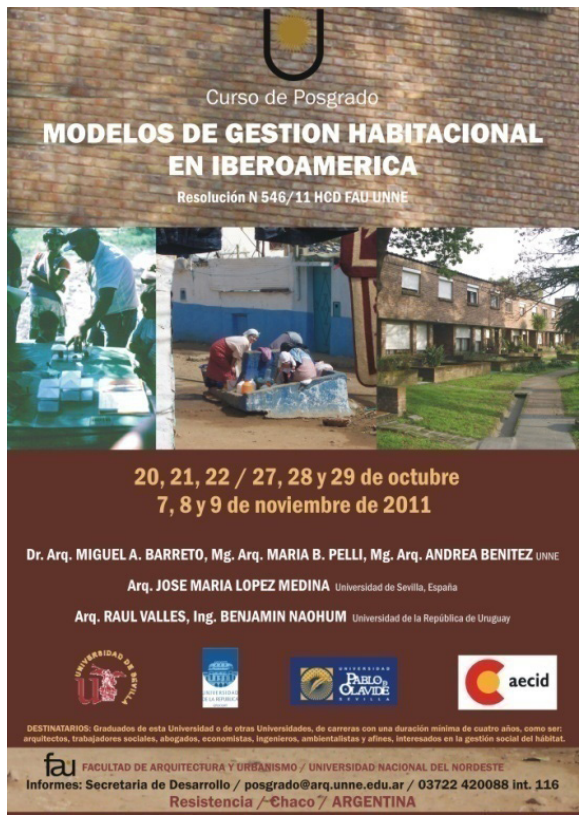

Figura 5. Cartel anunciador del curso de Posgrado Modelos de Gestión Habitacional en Iberoamérica. Fuente: IIDVI, UNNE.

3 Impartido por los docentes M. ${ }^{a}$ Bernabela Pelli, Miguel Ángel Barreto, M. ${ }^{\mathrm{a}}$ Andrea Benítez del equipo argentino de la UNNE, José M. ${ }^{\mathrm{a}}$ López Medina, de la Universidad de Sevilla, Raúl Vallés y Benjamín Nahoum de la UdelaR, con la coordinación académica de la UNNE, Resistencia, Chaco, Argentina, en octubre y noviembre de 2011. 


\section{MARCO TEORICO DE REFERENCIA PERSPECTIVA INTEGRAL y PARTICIPATIVA}

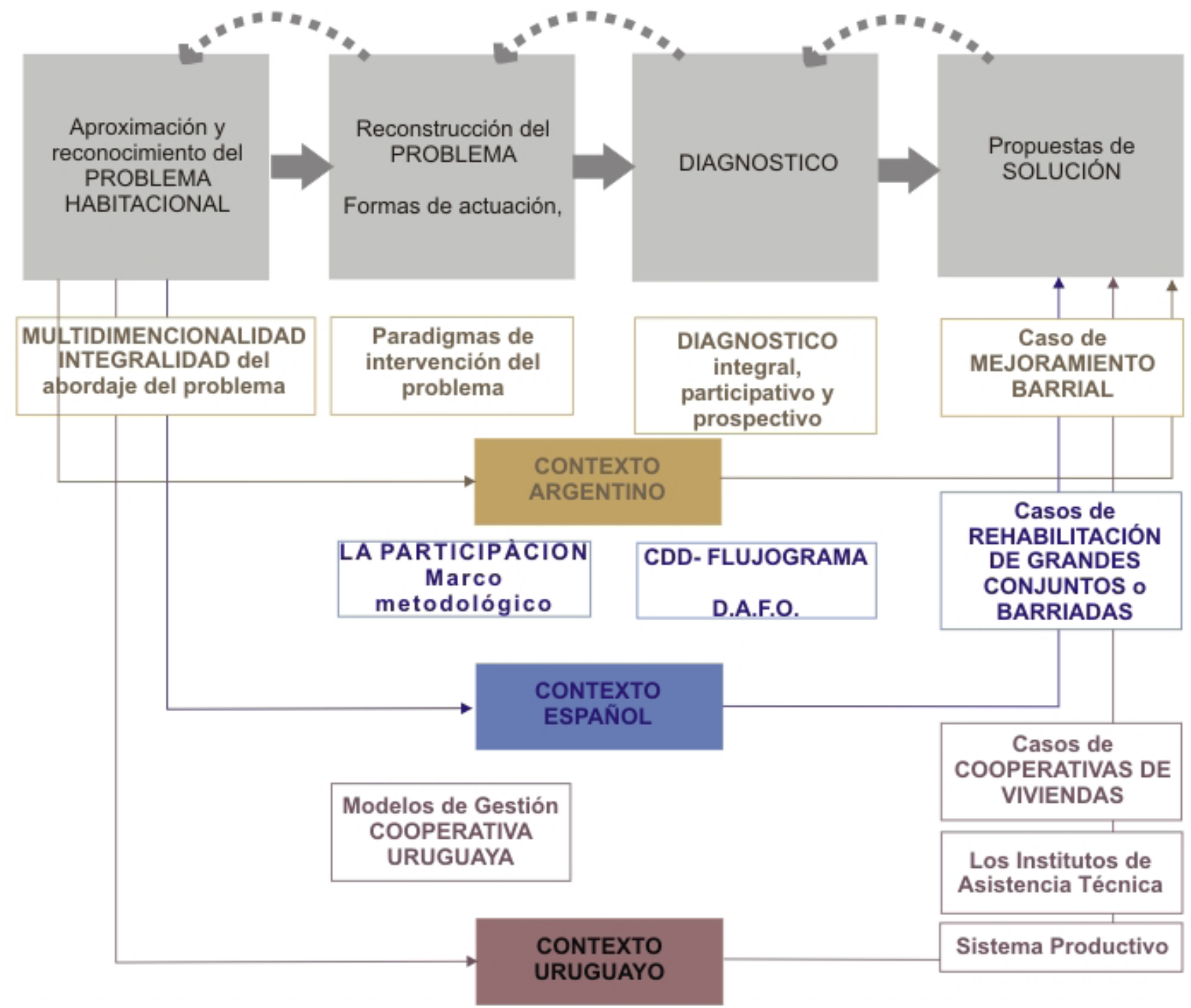

SINTESIS realizada por los cursantes

Reformulación del proyecto de intervención analizado en grupo durante el curso.

Elaboración de una nueva PROPUESTA DE INTERVENCIÓN, a partir de los contenidos incorporados en el curso

Figura 6. Diseño del curso de posgrado Modelos de Gestión Habitacional en Iberoamérica realizado en Resistencia. Fuente: Elaboración propia.
El valor de la oferta pedagógica, lo pudimos verificar en la respuesta de los participantes del curso, tanto en los trabajos finales, de gran calidad y profundidad, como en las valoraciones finales del curso. Valoraron positivamente el planteamiento del curso dirigido a profesionales implicados en su día a día en programas institucionales de hábitat ("El planteo del curso y la convocatoria realizada a las diferentes instituciones que se encuentran trabajando sobre la problemática habitacional, permitió una relación entre los grupos de trabajo de centros académicos donde se construyen conocimientos y los técnicos de los organismos mencionados."), la metodología ("Considero positiva la metodo- 

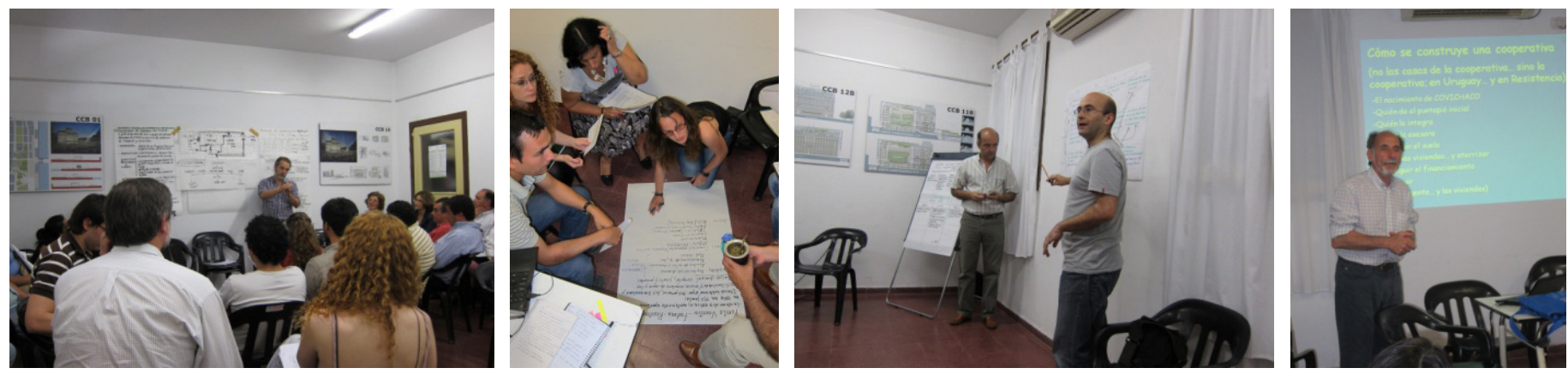

logía de trabajo planteada para el desarrollo del curso, la que alternaba entre clases teóricas y trabajos en taller, permitiendo poner en práctica los conceptos enunciados por los docentes, a la vez que el trabajo en grupo propició la participación y el intercambio de ideas, lo cual fue enriquecido por la convocatoria realizada a distintas disciplinas que permitió la conformación de equipos de trabajo multidisciplinarios.") y el propio hecho de constatar las similitudes de planteamientos y prácticas que los tres equipos docentes desarrollan en sus respectivos ámbitos geográficos ("Es destacable el diálogo que se genera en los tres módulos, a pesar de pertenecer a localidades muy lejanas y con procesos transitados muy diferentes, debido a que la producción social del hábitat en todos los casos ha cobrado la misma importancia y se expresa de manera muy similar, con modelos que incluyen la participación, la interdisciplina, la integración de múltiples actores y, sobre todo, la visión de un hábitat multidimensional, donde la realización de la vivienda es una pata que no se sostiene en el tiempo sin el aporte de las demás").

\section{Caso III. Salto en Salto}

El curso de Salto (Uruguay) significó de hecho un salto cualitativo en dos sentidos: en primer lugar, porque no surge como una oferta de la red, sino a raíz de una demanda institucional. Y en segundo lugar, porque se integraron los tres equipos en una única propuesta docente unitaria.

Se denominó "Herramientas para la Gestión participativa y la articulación interdisciplinar e interinstitucional en programas habitacionales de promoción pública". El curso estuvo especialmente dirigido a técnicos de organismos públicos del interior del país. Se organizaron los contenidos en dos módulos, el primero de Análisis de SituacionesProblema y el segundo sobre la Acción participativa, ambos dictados en junio del año 2012. La metodología de trabajo combinó las exposiciones teórico-metodológicas docentes, con instancias de debate y taller colectivos. Para aprobar el curso se requería la asistencia al $80 \%$ de las clases, así como la elaboración de un trabajo individual, consistente en un análisis-reflexión sobre la práctica profesional personal, en función de la temática, herramientas y metodologías que aportó el curso. Participaron 41 técnicos de 14 Departamentos del país. ${ }^{4}$

La valoración de los participantes a los aportes conceptuales del curso se refiere a la integralidad de la propuesta ("Me reconforta saber que desde el equipo docente se tiene una mirada integral hacia el sujeto y se toman en cuenta las dificultades que se presentan para una gran parte de la población en la adquisición de la vivienda"). Las posibilidades de reflexión sobre la propia práctica ("Me llevo la reflexión sobre temáticas con las cuales convivo en mi realidad laboral cotidiana. Reflexión que se refuerza y enriquece con los aportes teóricos del curso y
Figura 7. Universidad Nacional del Nordeste. Ciudad de Resistencia, Argentina, noviembre de 2011

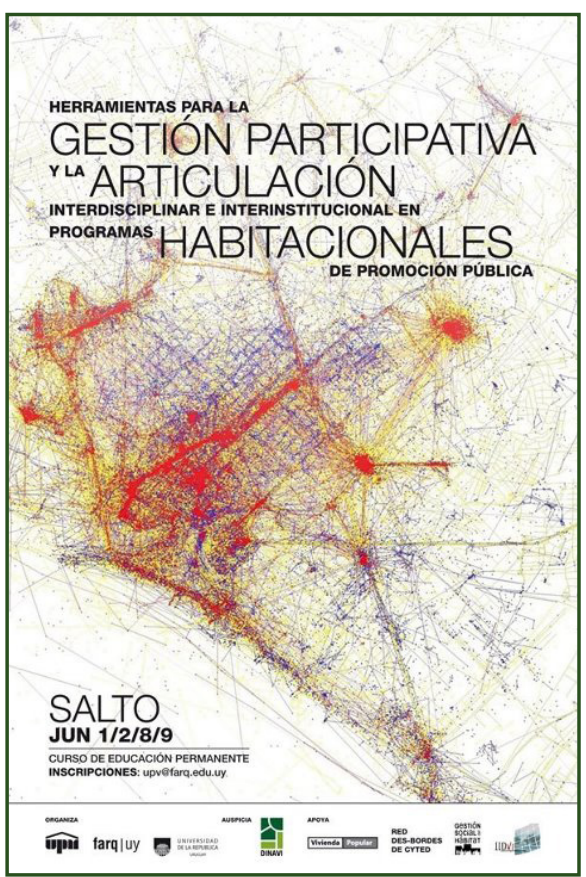

Figura 8: Cartel anunciador del curso de Postgrado en Salto. Fuente: Unidad Permanente de Vivienda (UPV) UdelaR.

4 La inserción institucional de los participantes fue la siguiente: MVOTMA-DINAVI, PIAI-PMB, algunos funcionarios del programa y otros técnicos que pertenecen a equipos técnicos que trabajan en asentamientos, otros de la Agencia Nacional de Vivienda (ANV), técnicos y políticos de distintas Intendencias e integrante de una organización social de Salto. Asimismo, los participantes eran de diversas disciplinas: agronomía, arquitectura, magisterio, psicología, trabajo social, tecnicatura en construcción. 
el intercambio entre los distintos participantes"). Se refieren también a la complejidad de la problemática y la necesidad de un abordaje multidimensional ("La importancia de la interdisciplina en todas las instituciones involucradas, trabajar más en el territorio y menos detrás de un escritorio").

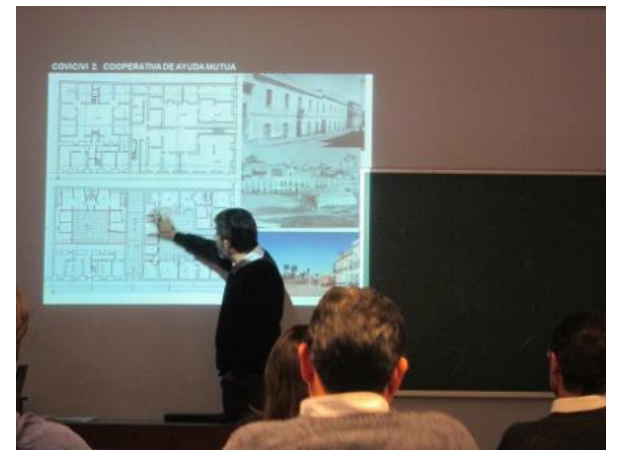

Figura 9. Curso de Herramientas para la Gestión Participativa y la Articulación interdisciplinar en programas habitacionales de promoción pública. Sede de la Regional Norte de UdelaR. Salto, Uruguay. 9 de junio de 2011.
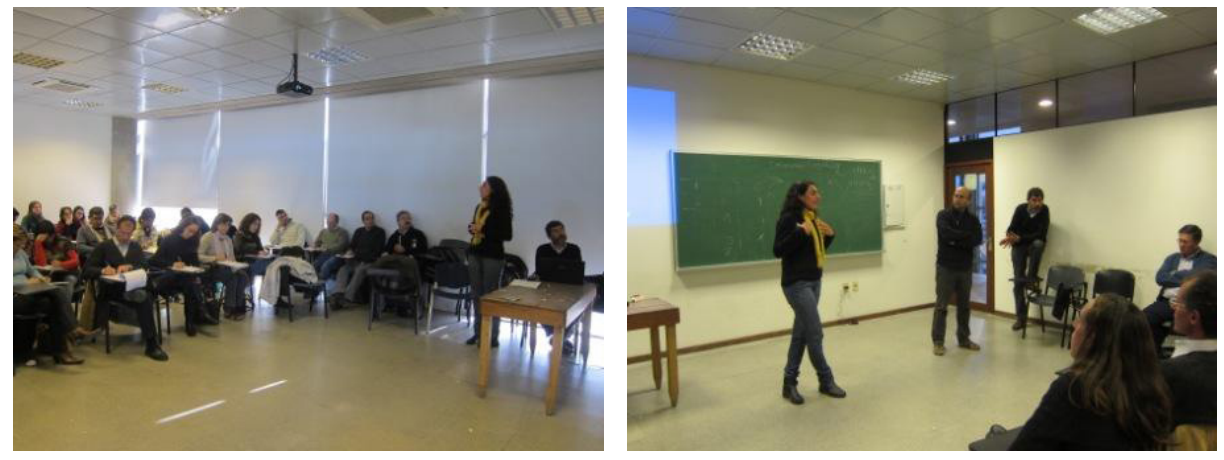

\section{Cantes de vuelta (2013-2016)}

Entre los años 2013 y 2016 cada equipo siguió su propia trayectoria formativa en posgrado en la que se recogen algunas derivadas de la experiencia cooperativa.

\section{Maestría en Resistencia}

En el año 2013 se puso en marcha la Maestría en Gestión y Desarrollo de la Vivienda Social, FAU UNNE. Motivados, por un lado, por el propio proceso de evolución del IIDVi y de la cátedra Gestión y Desarrollo de la Vivienda Popular (GDVP) de la carrera de Arquitectura, que han venido elaborando y acumulando experiencia creativa y conocimientos y por otro el nuevo impulso y experiencias que proporcionó el desarrollo conjunto de los cursos de posgrado, que aquí se mencionan.

El basamento teórico-ideológico que la sustenta presenta algunos de los rasgos característicos de la concepción del problema habitacional y de sus vías adecuadas de abordaje, sostenida tanto por el IIDVi como por este grupo de articulación internacional.

La primera edición se desarrolló con gran convocatoria de profesionales de la región del Nordeste Argentino (Provincias de Corrientes, Chaco, Formosa y Misiones), aproximadamente 50 estudiantes de diferentes disciplinas y procedencias laborales, pero la mayoría proveniente de los Institutos de Vivienda de la región. Actualmente se está editando la segunda cohorte de la Maestría, durante los años 2017 y 2018.

\section{Cursos intensivos en Andalucía}

La experiencia adquirida en los cursos organizados de forma cooperativa ha permitido al grupo ADICI adaptarse al contexto de crisis de España, que trajo aparejado el colapso de las políticas públicas de vivienda y rehabilitación, y reconvertir el máster en gestión social del hábitat (2008-2009 y 2009-2011) en cursos intensivos de menor duración con prácticas reales en terreno.

El primero de ellos, "Introducción a la Producción y Gestión Social del Hábitat. Co-Gestión de viviendas, locales y suelos públicos para ini- 
ciativas orientadas al bien común", fue un curso diseñado y ofertado a demanda de la empresa pública AVRA (Agencia de Vivienda y Rehabilitación de Andalucía). Tuvo formato semipresencial, apoyado en el Aula de Enseñanza Virtual de la Universidad de Sevilla, y se desarrolló en 2014. Hubo dos ediciones, una para Andalucía occidental y otra para Andalucía oriental, lo que permitió formar a los técnicos de la empresa de las ocho provincias andaluzas en materia de gestión social participativa, de cara a fomentar la puesta en uso de espacios y locales ociosos de su propiedad, en favor de iniciativas sociales orientadas al bien común. Fue impartido a trabajadores sociales, arquitectos, arquitectos técnicos, economistas y abogados de la empresa. Se formaron equipos de trabajo por territorios que aplicaron el curso al diseño de experiencias piloto reales tutorizadas por el equipo docente.

El segundo de ellos, "Curso de Formación Continua en Introducción a la Producción y Gestión social del Hábitat. Tecnologías apropiadas y apropiables (I edición)", Enseñanza Propia de la Universidad de Sevilla (14 ECTS) 2015/2016, es de alguna manera una reformulación intensiva del Máster en Gestión Social del Hábitat, así como una actualización de contenidos que aprovecha nuevos nichos profesionales para los gestores sociales del hábitat en torno a las Estrategias de Desarrollo Urbano Sostenible Integrado impulsadas por la Unión Europea. Se conjugaron contenidos en tres módulos: 1) avance de una estrategia barrial de sostenibilidad urbana integrada y diseño del proceso para su elaboración participada; 2) formulación de un proyecto de desarrollo local dentro de la estrategia; 3) diseño participativo de la reforma de una vivienda unifamiliar. El curso tuvo una duración de tres meses y un fuerte apoyo en la plataforma de enseñanza virtual de la Universidad de Sevilla, combinada con algunas sesiones presenciales con clientes reales. ${ }^{5}$ El resultado es que algunas de estas experiencias han desbordado el planteamiento académico y han tenido continuidad.

\section{Conclusiones}

\section{Sobre la demanda de formación en gestión compleja y participativa del hábitat}

La buena respuesta a los cursos (en cuanto a inscripciones, calidad de los trabajos, y evaluaciones positivas) permite constatar la demanda de formación en esta temática por parte de profesionales, investigadores, entidades y administraciones públicas en las distintas regiones donde se han impartido, con sus similitudes de contexto, pero también con sus obvias diferencias.

\section{Sobre la aplicabilidad y replicabilidad}

Constatar también la validez y utilidad, a partir del aprovechamiento que los grupos hacen en distintos contextos, del marco conceptual compartido y las herramientas teórico-metodológicas que se aportan, aplicadas a casos locales.

\section{Sobre el enfoque conceptual y metodológico}

Luego de transcurrir estas experiencias formativas de mayor convergencia entre los equipos, donde pudimos empezar a vislumbrar el es-
5 Para más información se puede consultar el libro memoria del curso (López Medina, De Manuel Jerez et al., 2016). 
quema conceptual referencial común que estábamos construyendo y a partir de las evaluaciones de los participantes de los cursos, pudimos detectar aspectos clave, que fortalecen la cooperación entre los equipos y la propuesta pedagógica común:

- Los estudiantes perciben un marco común de enfoque teórico metodológico de los tres equipos a partir de las experiencias transmitidas: validación del E.C.R.O. compartido sobre la base de "la participación, la interdisciplina, la integración de múltiples actores, y sobre todo la visión de un hábitat complejo multidimensional";

- La combinación de exposiciones teóricas con aplicación a casos reales en los que están involucrados los estudiantes funciona para producir aprendizajes significativos que son aplicados de forma inmediata a la propia práctica profesional que realizan en su ámbito laboral;

- La composición pluridisciplinar de los equipos de estudiantes que trabajan sobre una práctica común contribuye a la comprensión compleja del hábitat social y a la formación del propio equipo interdisciplinario;

- El intercambio entre académicos (docentes) y profesionales inmersos en la práctica de intervención en el hábitat (docentes y discentes) contribuye a crear una comunidad de aprendizaje donde todos aprendemos (docentes y discentes).

\section{Sobre el formato}

- Pudimos constatar, y comprobarlo en varios contextos regionales, que en un curso corto e intensivo es posible aportar claves que permiten analizar y reformular intervenciones;

- Apreciamos las ventajas e inconvenientes de la evolución hacia formatos más breves y flexibles (en el caso español, motivada por la coyuntura económica y laboral): pasar de un máster presencial de año y medio a la modalidad intensiva, online-semipresencial y 'deslocalizada'. Destacamos dentro de ese modelo la importancia de: la componente presencial y vivencial; el manejo de varias escalas de intervención; la posibilidad del intercambio entre varias ciudades/laboratorios de casos;

- Este formato intensivo de duración corta o media permite la participación en instancias formativas críticas de profesionales, en muchos casos pertenecientes a instituciones responsables de políticas de vivienda, que no pueden permitirse acceder a formaciones de mayor calado y largo recorrido;

- Estimamos, no obstante, que es igualmente deseable la existencia de una oferta de maestrías o posgrados expertos de mayor duración, en los que se pueda profundizar más en lo conceptual, teórico y metodológico como base para incidir a largo plazo en el fundamento ideológico y epistemológico de la formación de los profesionales que trabajan en la transformación del hábitat.

A partir de los resultados de las experiencias, del desarrollo del marco teórico compartido y de la evolución del planteo pedagógico de la oferta académica de posgrado a través de estos años, nos proponemos continuar fortaleciendo este espacio de cooperación. La red aspira, asimismo, a ampliar sus fronteras mediante nuevos desafíos de investiga- 
ción conjunta y de incorporación de nuevos equipos o administraciones. Nuevas idas y vueltas que permitirán profundizar en el mestizaje de esta milonga en construcción, esta cooperación docente que pretende contribuir a transformar los paradigmas de actuación para la mejora del hábitat humano. 


\section{Referencias}

Barreto, M.A., Benítez, M.A., López Medina, J.M. et al. (2011). Cooperación interuniversitaria de grupos latinoamericanos y españoles en docencia de posgrado. Dimensiones académicas y formativas de la producción y gestión social del hábitat. En Méndez, A., Salgado, M. y Vázquez Honorato, L. (comps.), Formación Universitaria en Hábitat. 20 años de Experiencias de la Red ULACAV. Red Universitaria Latinoamericana de Cátedras de Vivienda, pp. 251261.

De Manuel Jerez, E. (2002). Formar Arquitectos en la Universidad. En I Congreso de Educación para el Desarrollo en la Universidad. Valladolid: O.C.U.D.

- (2010). Construyendo triángulos para la gestión social del hábitat. Hábitat y Sociedad, 1, 13-37.

- (2017). Consolidación de Barrios. Caso de Jnane Aztout, Larache, Marruecos. En Pelli, V.S. et al. (orgs. y comps.), Experiencias Habitacionales Significativas en Latinoamérica y España. Resistencia: Editorial FAU UNNE, pp. 29-69.

De Manuel, E., Andrés Zambrana, L., Ojeda Rivera, J.F. et al. (2009). El Master en Gestión Social del Hábitat. XV Encuentro Internacional de la Red Ulacav. Cátedra de Gestión y Desarrollo de la Vivienda Popular. Resistencia-Chaco-Argentina, 15, 1-20. ISBN: Recuperado el 27 de marzo de 2017 de: http:/ /www.redulacav.org/material2009.php.
Gasca-Pliego E. y Olvera-García J. (2011). Construir ciudadanía desde las universidades, responsabilidad social universitaria y desafíos ante el siglo xxI. Convergencia, 18 (56), 37-58.

López Medina, J. M ${ }^{\mathrm{a}}$, De Manuel Jerez, E., Iglesias Onofrio, M. et al. (coords.) (2016). Hábitat en proceso. Memoria del curso Introducción a la Producción y gestión social del hábitat. Sevilla: GSH.

Lucca, E. (2014). Interdisciplina. Práctica esencial para el abordaje social y ambiental. Resistencia: Ediciones Arbres de Vie, Cospel.

Morales, P. y Landa, V. (2004). Aprendizaje basado en problemas. Theoria, 13 (1), 145-157.

Morin, E. (1999). La cabeza bien puesta: repensar la reforma, reformar el pensamiento. Buenos Aires: Nueva Visión.

Pelli, V. S. (2010). La gestión de la producción social del hábitat. Hábitat y Sociedad, 1, 39-54.

Pelli, V.S., Alcalá, L.I., Giró M.G. et al. (2015). Maestría en Gestión y Desarrollo de la Vivienda Social de la Facultad de Arquitectura y Urbanismo de la UNNE. Gestación y evolución. Revista ADNea. Arquitectura y Diseño del Nordeste argentino, 3 (3), 43-50.

Shön, D. (1992). La formación de profesionales reflexivos: hacia un nuevo diseño de la enseñanza y el aprendizaje en las profesiones. Barcelona: Centro de Publicaciones del Ministerio de Educación y Ciencia.

De Manuel Jerez, E., Pelli, M. B. y López Medina, J. M. (2017). Cantes de ida y vuelta en la formación sobre la gestión del hábitat. Aprendizajes y confluencias entre el Paraná y el Guadalquivir. Hábitat y Sociedad, 10, 323-334.

<http://dx.doi.org/10.12795/HabitatySociedad.2017.i10.18>

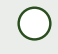

